\title{
Low Frequency EPR and EMR Point Spectroscopy and Imaging of a Surface
}

\author{
Lauren E. Switala ${ }^{1}$, William J. Ryan ${ }^{1}$, Merlin Hoffman ${ }^{2}$, Wyatt Brown ${ }^{1}$, Joseph P. Hornak ${ }^{1}$ \\ ${ }^{1}$ Magnetic Resonance Laboratory \\ Rochester Institute of Technology \\ Rochester, NY 14623-5604 \\ ${ }^{2}$ Oberlin College \\ Oberlin, $\mathrm{OH} 44074$
}

\author{
Corresponding Author \\ Joseph P. Hornak \\ Magnetic Resonance Laboratory \\ 54 Lomb Memorial Drive, 76-2132 \\ Rochester Institute of Technology \\ Rochester, NY 14623-5604 \\ jphsch@rit.edu \\ $585475-2904$
}




\begin{abstract}
Low frequency electron paramagnetic resonance (LFEPR) spectrometers operating between 100 and $500 \mathrm{MHz}$ typically have large-volume magnets that accommodate large samples. LFEPR spectroscopy with a $2.9 \mathrm{~mm}$ diameter surface coil was used to record point spectra and image the spatial distribution of the spin probe 2,2-Diphenyl-1-picrylhydrazyl (DPPH) and electrophotographic toner in printed letters on a flat surface. The location of the surface coil was fixed on the desired location when a spectrum was recorded. The magnetic field of the spectrometer was fixed on the location of the signal and the sample was scanned under the surface coil in parallel trajectories to produce an image of the signal in the letters "LFEPR". We speculate on the utility of this technique to study flat objects such as paintings and illuminated manuscripts with cultural heritage significance.
\end{abstract}

Key Words: Low frequency electron paramagnetic resonance, LFEPR, electron paramagnetic resonance, EPR, electron magnetic resonance, EMR, surface imaging, toner, DPPH 


\section{Introduction}

Electron paramagnetic resonance (EPR) and electron magnetic resonance (EMR) spectroscopies are commonly performed at Larmor frequencies $(v)$ at or above $9 \mathrm{GHz}$ and restricted to samples of a few $\mathrm{mm}^{3}$ in volume. Examination of large, intact $(>10 \mathrm{~cm})$ objects with EPR spectroscopy is challenging owing to the limitations of cavity resonators used for providing the oscillating magnetic field $\left(\mathrm{B}_{1}\right)$ at the Larmor frequency to the sample and the small space between the poles of the magnet producing the applied magnetic field ( $\left.\mathrm{B}_{\mathrm{o}}\right)$. A $9 \mathrm{GHz} \mathrm{EPR}$ design that has the potential to examine larger, intact objects has been reported, [1] however it was developed to examine smaller, near-microscopic $\left(600 \mu \mathrm{m}^{3}\right)$ objects.

Low frequency electron paramagnetic resonance (LFEPR) spectrometers typically operate at $100<v<500 \mathrm{MHz}$ and can accommodate samples which are several liters in volume.[2-5] The ability to accommodate large samples is attributed to the large volume magnetic field [3-5] and the lower operating frequency of LFEPR spectrometers compared to conventional $9 \mathrm{GHz}$ EPR spectrometers. The lower operating frequency makes the construction of surface coils and creation of large volume sample probes simpler.

LFEPR spectroscopy also avoids microwave heating of tissues associated with $9 \mathrm{GHz} \mathrm{EPR}$ and can therefore be used in whole animal or human studies. [4,5] This paper focuses on a materials application of LFEPR, although the techniques we present may have applicability in vivo studies.

EPR has been used to study objects with cultural heritage significance by destructively or invasively removing a small part of a large object and placing it in a spectrometer.[6-11] LFEPR, however, can be used to study large objects with cultural heritage significance noninvasively and non-destructively.[2] In this paper we address the utility of LFEPR to study flat 
objects containing paramagnetic or ferri/ferromagnetic pigments by demonstrating the ability of LFEPR to both record point spectra and image the spatial distribution of the stable free radical 2,2-Diphenyl-1-picrylhydrazyl (DPPH) and electrophotographic toner. Several electrophotographic toners contain ferri/ferromagnetic nanoparticles.[12,13] The EMR g factor of these nanoparticles tends to be smaller than for atomic ions and molecular radicals as the magnetic domains are larger. The $\mathrm{g}$ factor for toner is approximately $1.85,[13]$ while that for molecular radicals and ionic atoms is greater than two.

\section{Materials and Methods}

We used the laboratory built continuous wave LFEPR spectrometer with magnetic field modulation and phase sensitive detection at $10 \mathrm{kHz}$ described previously.[2,3] The field modulation was provided by a $15 \mathrm{~cm}$ inside diameter (ID) Helmholtz coil driven by a Techron 7570 audio frequency amplifier. The sample resonator was a $2.9 \mathrm{~mm}$ ID inductively coupled surface coil (SC) consisting of a closely wound, five-turn solenoid of \#26 enameled copper wire and a $5 \mathrm{pF}$ chip capacitor. The SC had a resonance frequency (v) of $271.46 \mathrm{MHz}$ and a quality factor $(\mathrm{Q})$ of 75 . When the surface coil is placed against a surface, its radio frequency magnetic field $\left(B_{1}\right)$ is perpendicular to the surface. The $S C$ was fixed in the center of the $30 \mathrm{~cm}$ ID solenoidal electromagnet such that $\mathrm{B}_{1}$ was perpendicular to the $\mathrm{B}_{\mathrm{o}}$ magnetic field from the electromagnet. $B_{0}$ could be swept or fixed at any value between 0 and $18.7 \mathrm{mT}$. For surface spectroscopy or imaging, the sample is located on an acrylic sample stage that was moved under the $\mathrm{SC}$ with a two axis positioning system located outside the magnet.

The sensitivity map of the surface coil defines the sample volume for point spectroscopy and the best point spread function (PSF) achievable for surface imaging. An approximate 
relative sensitivity map of the surface coil in a plane perpendicular to the circular edge of the coil and passing through the diameter was measured and is presented in Fig. 1. The sensitivity was mapped by moving a $1.5 \mathrm{~mm}$ diameter, $0.25 \mathrm{~mm}$ thick disc of DPPH in epoxy by $0.02 \mathrm{~mm}$ steps using a two-axis micrometer.

Rotating the two-dimensional sensitivity map about the SC axis gives an approximation of the three-dimensional sensitivity map $(\mathrm{S}(\mathrm{x}, \mathrm{y}, \mathrm{z}))$ for the SC. The SC sensitivity drops off with distance from the coil center. In general, the sensitive region of the SC can be thought of as spherical cap on the bottom of the coil. If 0.34 is arbitrarily chosen as the minimum, useful, relative signal value, a $3.8 \mathrm{~mm}^{3}$ sensitive volume for the coil is defined by a spherical cap of height $0.75 \mathrm{~mm}$, radius $2.25 \mathrm{~mm}$, and base radius $1.75 \mathrm{~mm}$. When the $\mathrm{SC}$ is placed on a sample with a concentration distribution of spins, $\mathrm{C}(\mathrm{x}, \mathrm{y}, \mathrm{z})$, the EPR signal is proportional to the volume integral of the product of $\mathrm{C}(\mathrm{x}, \mathrm{y}, \mathrm{z})$ and $\mathrm{S}(\mathrm{x}, \mathrm{y}, \mathrm{z})$.

$$
\text { EPR Signal } \propto \iiint C(x, y, z) S(x, y, z) d x d y d z
$$

In the limit of infinitely thin samples, the volume integral becomes an area integral and the sensitive region becomes a circular disc.

It should be noted that the sensitivity map of Fig. 1. only approximates the PSF owing to the finite size of the DPPH disc used to create the sensitivity map. For a $0.25 \mathrm{~mm}$ thick surface sample, the PSF as a function of radial position (r) of the coil is approximately

$$
P S F=-0.136 r^{2}-0.187 r+0.998
$$

Two samples were used to test the performance of the point spectroscopy and surface imaging capabilities of the system. The first was DPPH, which has a single $g=2.0036$ peak in the EPR spectrum. This sample was prepared by mixing DPPH (Sigma-Aldrich) in epoxy (Loctite, $5 \mathrm{~min}$ ) and stenciling \#72 Arial font (18.3 $\mathrm{mm}$ high) letters LFEPR in a $0.2 \pm 0.05 \mathrm{~mm}$ 
thick layer on a sheet of vinyl. (See Fig. 2.) Note that these stenciled letters are not perfect: the letters $\mathrm{F}$ and $\mathrm{E}$ have a slight tilt to the left, and there are discontinuities where the bowl of the $\mathrm{P}$ and $\mathrm{R}$ attach to the stem due to filling the gap created by the bridge supporting the island in the letters' bowl with epoxy/DPPH.

The electrophotographic toner sample was a piece of $75 \mathrm{~g} / \mathrm{m}^{2}$ weight, 92 bright, white recycled copy paper (Staples ${ }^{\circledR}$ 620016) with the letters LFEPR printed on it in \#72 Arial font by a Hewlett-Packard $^{\circledR}$ (HP) LaserJet 1200 printer using OfficeMax ${ }^{\circledR}$ toner formulated for this HP printer. The EMR linewidth $(\Gamma)$ of ferri/ferromagnetic nanoparticles is dependent on the size and shape of the nanoparticle, as well as interparticle interactions. [14,15] The 9.1 GHz EMR spectrum of HP toner has a $\mathrm{g} \approx 1.85, \Gamma \approx 110 \mathrm{mT}$ absorption. [13] This $\Gamma$ value is greater than the $\mathrm{B}_{0}=10.47 \mathrm{mT}$ value for $\mathrm{g}=1.85$ at $\nu=271 \mathrm{MHz}$, as well as the sweep width on our instrument.

\section{Results}

Spectra from a point in each sample were obtained by centering the probe on the stem of the letter L. Fig. 3a shows the LFEPR spectra of DPPH in epoxy and for the paper. The LFEPR spectrum of toner fused to paper has the characteristic feature displayed in Fig. 3b. The signal increases from a minimum at $\mathrm{B}_{0}=0$ as $\mathrm{B}_{0}$ is increased, similar to that seen for some ferromagnetic nanoparticles at $9.5 \mathrm{GHz}$. [16] In the absence of toner on the paper, the signal is equal to the background noise of the instrument.

For mapping the spatial distribution of DPPH and toner in our text samples, the magnetic field was fixed at the value $\mathrm{B}_{\mathrm{F}}$ indicated by the vertical lines in Fig. 3., and the LFEPR spectrometer set to constantly record signal at a rate of 5 points/s with an $\mathrm{RC}$ time constant of 0.1

s. The sample was moved at $1 \mathrm{~mm} / \mathrm{s}$ so as to acquire signal along $\mathrm{n}$ parallel trajectories running 
the length of the sample and separated by $\Delta$. The $\mathrm{n}$ subsequent signal versus distance spectra were processed with a linear baseline correction, then combined to form a $561 \times \mathrm{n}$ element matrix. No additional processing was performed on this matrix to form the image composed of $0.2 \times \Delta$ mm pixels. The $\mathrm{B}_{\mathrm{F}}, \mathrm{n}$, and $\Delta$ values for $\mathrm{DPPH}$ and toner were respectively $9.55 \mathrm{mT}, 30,1 \mathrm{~mm}$ and $7.5 \mathrm{mT}, 17,2 \mathrm{~mm}$. As there is no peak in the EMR spectrum of toner on paper, we arbitrarily choose $\mathrm{B}_{\mathrm{F}}=7.5 \mathrm{mT}$, where the signal-to-noise ratio equals 10 , to record the signal. Fig. 4 displays the $561 \times 30$ pixel image for DPPH (a) and the $561 \times 17$ pixel image for toner (b).

\section{Discussion}

Figure 3 demonstrated that the SC, positioning system, and LFEPR spectrometer can be used to record an EPR point spectrum of a stable free radical and the smaller EMR signal from electrophotographic toner fused to the surface of a sheet of paper. Our current LFEPR spectrometer can accommodate a $15 \mathrm{~cm}$ wide, $3 \mathrm{~m}$ long flat object. The former limit is set by the modulation coil diameter while the latter is set by the room dimensions. The spatial resolution is limited by the diameter of the SC and the spatial sampling. Spectral resolution is limited by the typical EPR spectral parameters such as $B_{o}$ homogeneity and modulation amplitude. We believe this ability will allow us to place large flat objects such as illuminated manuscripts and paintings in our magnet and identify the composition of a pigment based on its EPR spectrum. The spectrometer may also be able to distinguish between pigments with similar colors but different EPR spectral features, as was demonstrated previously in a single turn solenoid volume resonator for Han, Egyptian, and ultramarine blue pigments.[2]

Owing to the $3.5 \mathrm{~mm}$ diameter of the sensitive region under the $\mathrm{SC}$, both text samples were oversampled in the length direction at $5 \mathrm{pts} / \mathrm{mm}$. The $\Delta$ increment between the scan lines was 1 $\mathrm{mm}$ for DPPH and $2 \mathrm{~mm}$ for toner. The large $\Delta$ for toner was chosen for expediency and to 
determine the effect of the large value on quality. Some detail was lost in the text quality but the letters were clearly visible. The quality of the displayed images could have been improved by interpolating in the $\mathrm{n}$ direction to achieve square pixels, but this was not performed so as to give a true representation of the capabilities of the technique. Note that the stenciling process did not produce a uniform distribution of DPPH in the letters, and that the letters F and E were at a slight angle. The imaging was able to pick up this slight angle in the two letters.

\section{Conclusions}

Our results demonstrate the utility of LFEPR with a small diameter surface coil to measure the spatial distribution of an EPR or EMR signal-containing material on a large flat surface. This result cannot be achieved with conventional, high frequency EPR spectrometers which utilize iron core magnets with narrow pole gaps as it is extremely difficult to satisfy the resonance condition of $\mathrm{B}_{1}$ perpendicular to $\mathrm{B}_{\mathrm{o}}$ with this geometry. With the larger air core electromagnets found on LFEPR spectrometers it is easier to satisfy the resonance condition and perform raster based surface imaging with a small surface coil. Our results open up the possibility of spectral imaging of multiple components in a painting or illuminated manuscript by either choosing $\mathrm{B}_{\mathrm{o}}$ values corresponding to different spectral peaks or scanning an entire LFEPR spectrum for a grid of locations in the sample.

\section{Acknowledgement}

This work was supported in part by an RIT College of Science Dean's Research Initiation Grant. MH acknowledges support from the National Science Foundation REU Program grant PHY1359361. 


\section{References}

1. J. P. Campbell, J.T. Ryan, P. R. Shrestha, Z. Liu, C. Vaz, J.-H. Kim, V. Georgiou, K. P. Cheung, Electron Spin Resonance Scanning Probe Spectroscopy for Ultrasensitive Biochemical Studies. Anal. Chem. 87 4910-4916 (2015).

2. W.J. Ryan, N. Zumbulyadis, J.P. Hornak, The Potential of Low Frequency EPR Spectroscopy in Studying Pottery Artifacts and Pigments, MRS Proceedings, mrsf13-1656pp03-03 doi:10.1557/opl.2014.708 (2015).

3. J.P. Hornak, M. Spacher, R.G. Bryant, A Modular Low Frequency ESR Spectrometer, Meas. Sci. Technol. 2 (1991) 520-522. DOI: 10.1088/0957-0233/2/6/005

4. G.A. Rinard, R.W. Quine, S.S. Eaton, G.R. Eaton, E.D. Barth, C.A. Pelizzari, H.J. Halpern, Magnet and Gradient Coil System for Low-Field EPR Imaging, Magn. Reson. Engineer. 15 (2002) 51-58. DOI: $10.1002 / \mathrm{cmr} .10018$

5. I. Salikhov, T. Walczak, P. Lesniewski, N. Khan, A. Iwasaki, R. Comi, J. Buckey, H.M. Swartz, EPR Spectrometer for Clinical Applications, Magn. Reson. Med. 54 (2005)13171320. DOI: $10.1002 / \mathrm{mrm} .20689$

6. G. V. Robins, N. J. Seeley, D. A. C. McNeil, M. R. C. Symons, "Identification of ancient heat treatment in flint artefacts by ESR spectroscopy," Nature 276, 703-704 (1978).

7. D. Cordischi, D. Monna, A. Segre, "ESR analysis of marble samples from Mediterranean quarries of archaeological interest," Archaeometry 25, 68-76 (1983).

8. D. Attanasio, D. Capitani, C. Federici, A. Segre, "Electron spin resonance study of paper samples dating from the fifteenth to the eighteenth century," Archaeometry 37, 377-384 (1995). 
9. T. Warashina, T. Higashimura, Y. Maeda, "Determination of the firing temperature of ancient pottery by means of ESR spectrometry," in Scientific studies in ancient ceramics(British Museum Research Laboratory, 1981), pp. 117-128.

10. Y. Bensimon, B. Deroide, S. Clavel, J.-V. Zanchetta, "Electron spin resonance and dilatometric studies of ancient ceramics applied to the determination of firing temperature," Japanese journal of applied physics 37, 4367 (1998).

11. F. Presciutti, D. Capitani, A. Sgamellotti, B. G. Brunetti, F. Costantino, S. Viel, A. Segre, "Electron paramagnetic resonance, scanning electron microscopy with energy dispersion Xray spectrometry, X-ray powder diffraction, and NMR characterization of iron rich fired clays," The Journal of Physical Chemistry B 109, 22147-22158 (2005).

12. J.P. Hornak, MRI of Printed Text, Concepts Magn. Reson. 36A (2010) 347-348. DOI: 10.1002/cmr.a.20169

13. N.N. Lobanov, V.N. Nikiforov, S.A. Gudoshnikov, V.P. Sirotinkin, Yu.A. Koksharov, N.A. Usov, V.G. Sredin, Yu.S. Sitnov, A.V. Garshev, V.I. Putlyaev, D.M. Itkis, O.A. Skoromnikova, G.N. Fedotov, Differentiation of Magnetic Composites in Terms of Their Nanostructural Organization, Doklady Chem. 426 (2009) 96-100.

$10.1134 / \mathrm{S} 0012500809050024$

14. R. Berger, J. Kliava, J.C. Bissey, V. Baietto, Magnetic Resonance of Superparamagnetic Iron-Containing Nanoparticles in Annealed Glass, J. Appl. Phys. 87 (2000) 7389-7396.

15. R. Berger, J.C. Bissey, J. Kliava, H. Daubric, C. Estournes, Superparamagnetic Resonance of Ferric Ions in Devitrified Borate Glass, J. Magn. Magn. Mater. 234 (2001) 535-544. 
16. R.S. de Biasin, R.D. de Souza Lopes, D.G. Carvalho, A.B.-H. da Silva Figueiredo, FMR Lineshape of Cobalt Ferrite Nanoparticles, Ceramics International, 41(2015) 865-867. DOI: 10.1016/j.ceramint.2014.08.129

\section{Figure Captions}

Fig. 1. The measured EPR sensitivity map for a plane perpendicular to the bottom the coil. The two circles indicate the position of the bottom winding of the surface coil.

Fig. 2. Images of the DPPH in epoxy stenciled on vinyl (top), and electrophotographic toner fused to paper (bottom).

Fig. 3. The LFEPR spectra of a) DPPH in epoxy, and b) electrophotographic toner on paper and paper without toner. Vertical line indicates the signal at the $\mathrm{B}_{\mathrm{o}}$ value used to scan the images in Fig. 2.

Fig. 4. An LFEPR image of the \#72 font letters LFEPR stenciled in epoxy with DPPH on a sheet of vinyl (top), and electrophographically printed toner on paper (bottom). The images were obtained by rastering the flat sample under a small surface coil probe while keeping $\mathrm{B}_{\mathrm{o}}$ fixed at respectively 9.55 and $7.5 \mathrm{mT}$. 


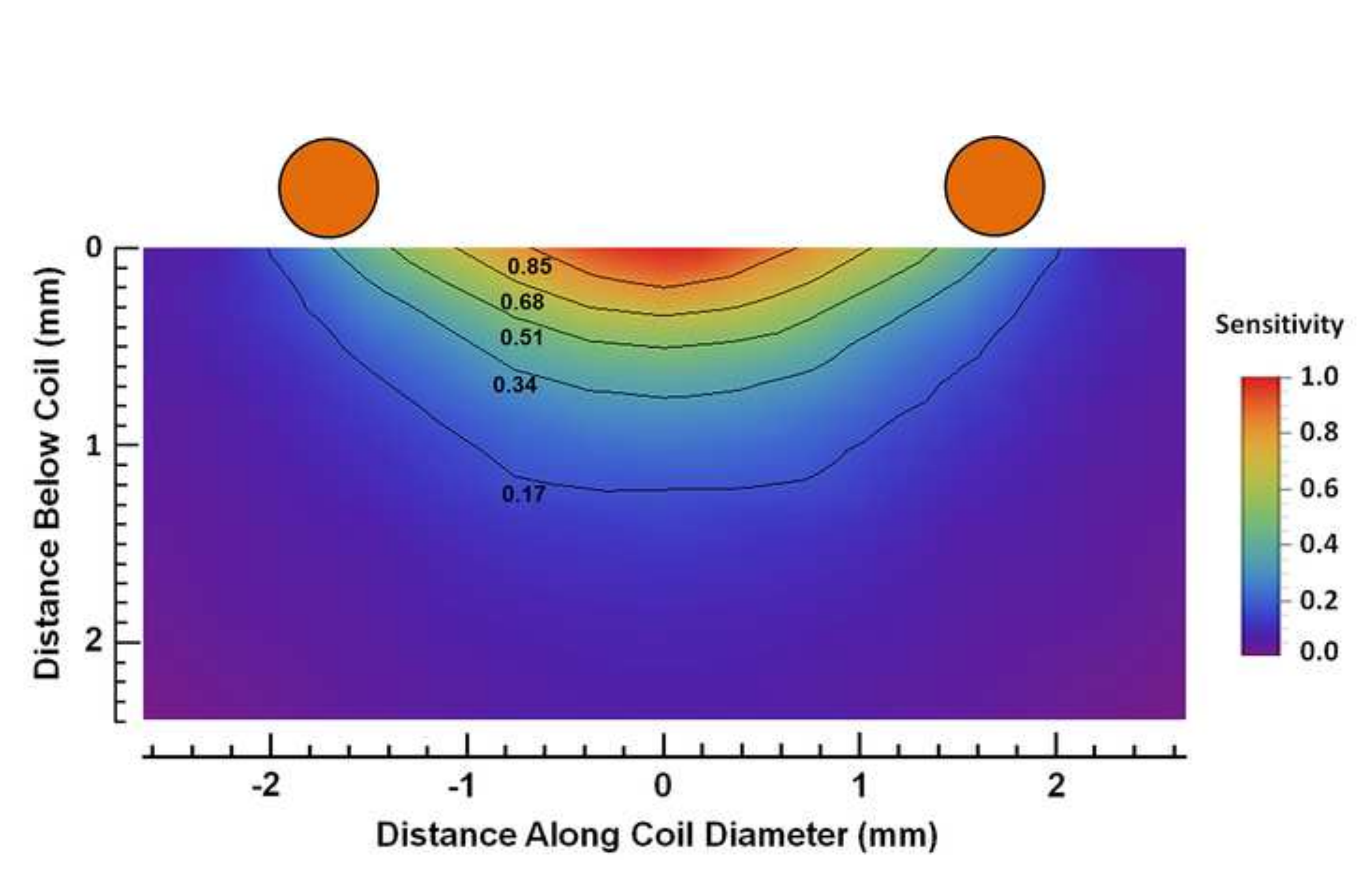

$\begin{array}{r}0.8 \\ 0 \\ 0.2 \\ 0 \\ 0.2 \\ \hline\end{array}$
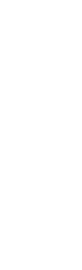

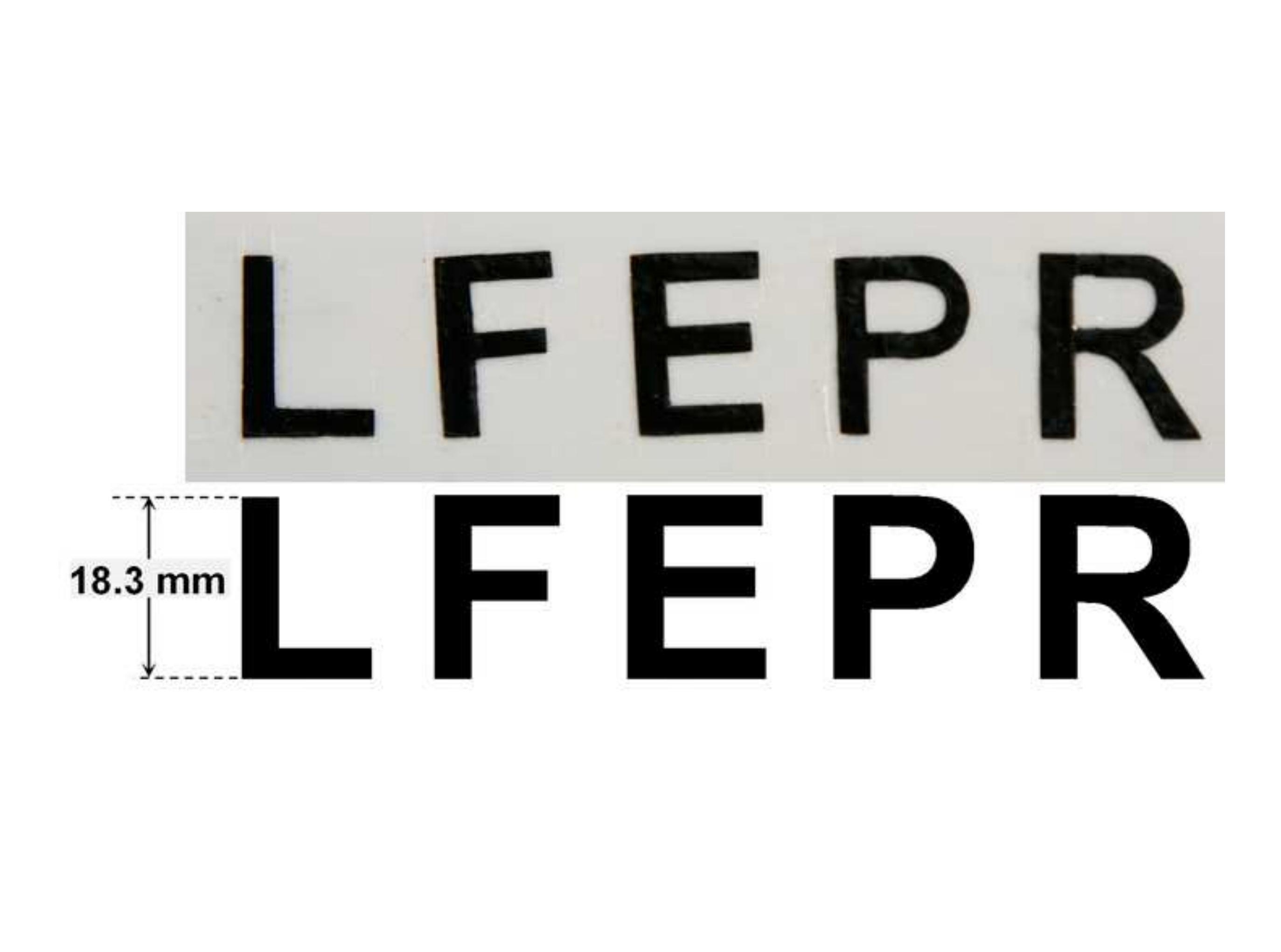


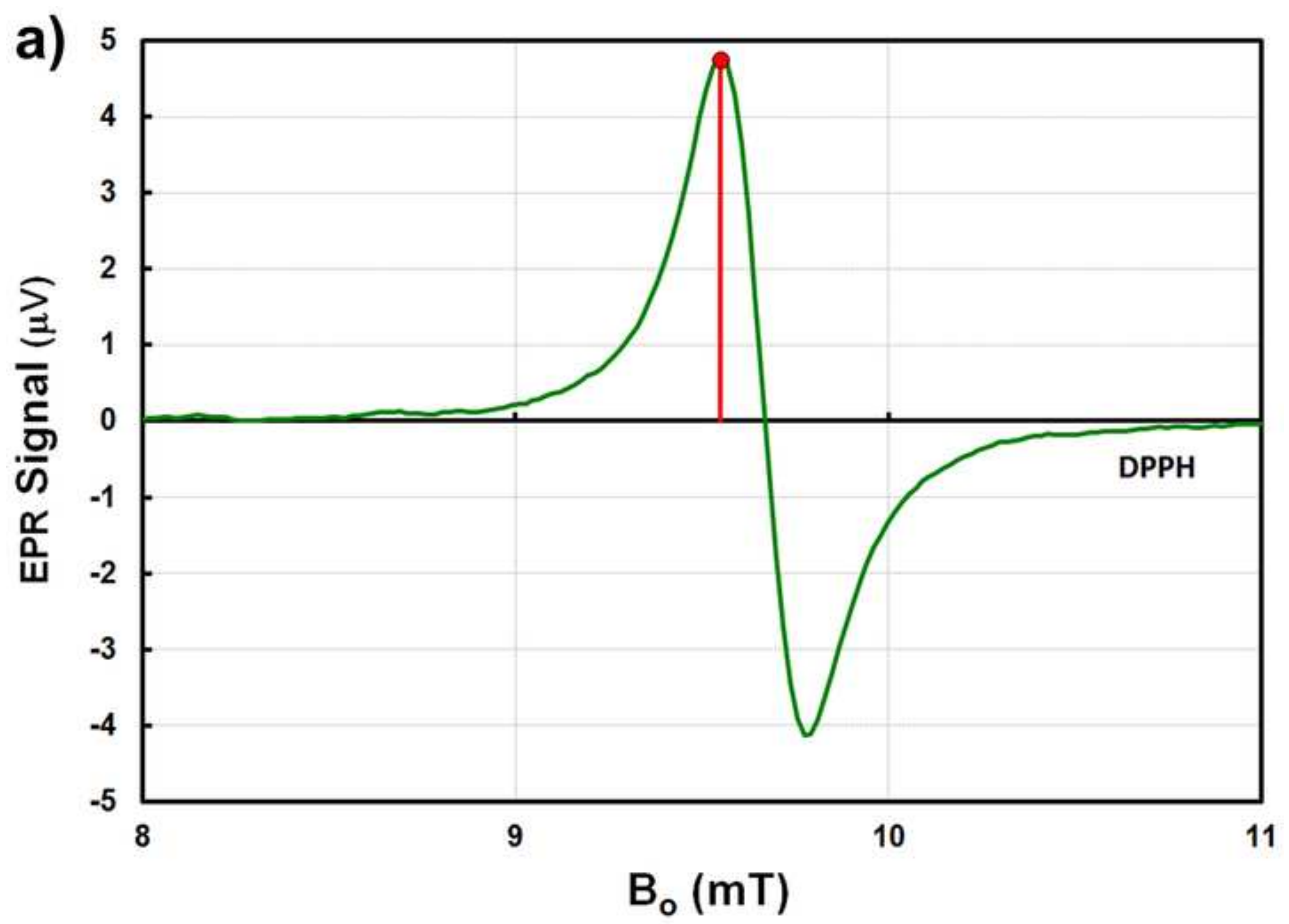

1




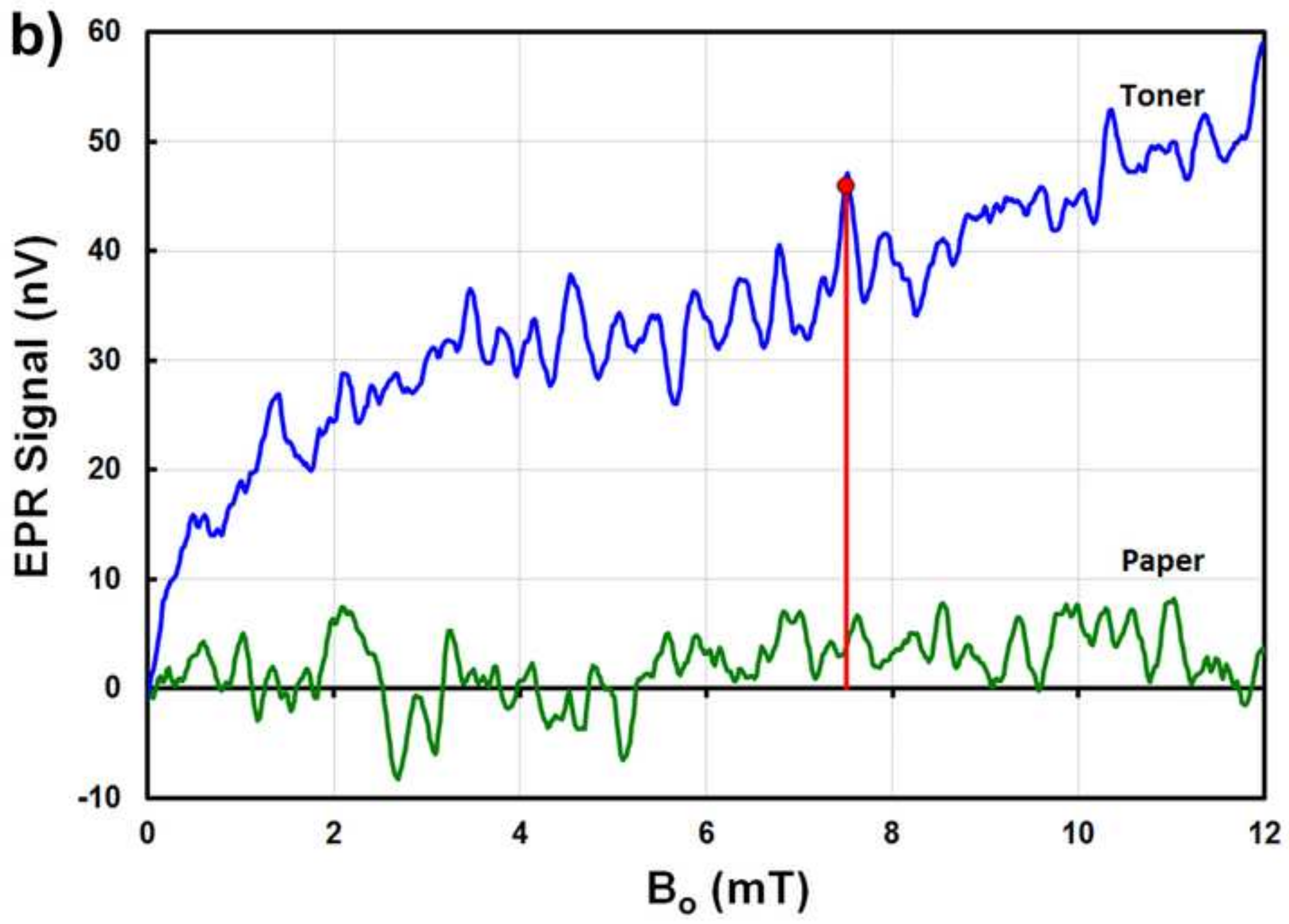


